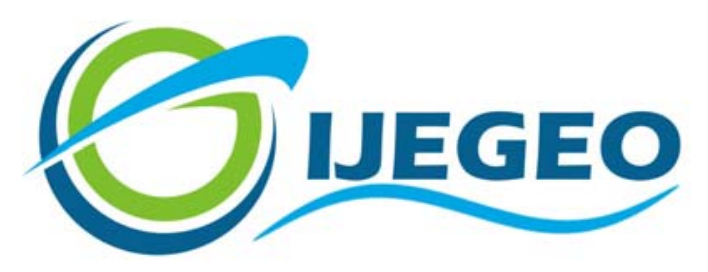

International Journal of Environment and Geoinformatics (IJEGEO) is an international, multidisciplinary, peer reviewed, open access journal.

\title{
A novel approach to automatic detection of interest points in multiple facial images
}

\author{
Bülent Bayram, G. Çiğdem Çavdaroğlu, Dursun Zafer Şeker, Sıtkı Külür
}

\section{Editors}

Prof. Dr. Cem Gazioğlu, Prof. Dr. Dursun Zafer Şeker, Prof. Dr. Ayşegül Tanık, Assoc. Prof. Dr. Şinasi Kaya

\section{Scientific Committee}

Assoc. Prof. Dr. Hasan Abdullah (BL), Assist. Prof. Dr. Alias Abdulrahman (MAL), Assist. Prof. Dr. Abdullah Aksu, (TR); Prof. Dr. Hasan Atar (TR), Prof. Dr. Lale Balas (TR), Prof. Dr. Levent Bat (TR), Assoc. Prof. Dr. Füsun Balık Şanlı (TR), Prof. Dr. Nuray Balkıs Çağlar (TR), Prof. Dr. Bülent Bayram (TR), Prof. Dr. Şükrü T. Beşiktepe (TR), Dr. Luminita Buga (RO); Prof. Dr. Z. Selmin Burak (TR), Assoc. Prof. Dr. Gürcan Büyüksalih (TR), Dr. Jadunandan Dash (UK), Assist. Prof. Dr. Volkan Demir (TR), Assoc. Prof. Dr. Hande Demirel (TR), Assoc. Prof. Dr. Nazlı Demirel (TR), Dr. Arta Dilo (NL), Prof. Dr. A. Evren Erginal (TR), Dr. Alessandra Giorgetti (IT); Assoc. Prof. Dr. Murat Gündüz (TR), Prof. Dr. Abdulaziz Güneroğlu (TR); Assoc. Prof. Dr. Kensuke Kawamura (JAPAN), Dr. Manik H. Kalubarme (INDIA); Prof. Dr. Fatmagül Kılıç (TR), Prof. Dr. Ufuk Kocabaş (TR), Prof. Dr. Hakan Kutoğlu (TR), Prof. Dr. Nebiye Musaoğlu (TR), Prof. Dr. Erhan Mutlu (TR), Assist. Prof. Dr. Hakan Öniz (TR), Assoc. Prof. Dr. Hasan Özdemir (TR), Prof. Dr. Haluk Özener (TR); Assoc. Prof. Dr. Barış Salihoğlu (TR), Prof. Dr. Elif Sertel (TR), Prof. Dr. Murat Sezgin (TR), Prof. Dr. Nüket Sivri (TR), Assoc. Prof. Dr. Uğur Şanlı (TR), Assoc. Prof. Dr. Seyfettin Taş (TR), Assoc. Prof. Dr. İ. Noyan Yılmaz (TR), Assist. Prof. Dr. Baki Yokeş (TR), Assist. Prof. Dr. Sibel Zeki (TR), Dr. Hakan Kaya (TR). 


\title{
A novel approach to automatic detection of interest points in multiple facial images
}

\author{
Bülent Bayram 1,"* G. Çiğdem Çavdaroğlu², Dursun Zafer Şeker ${ }^{3}$ and Sıtkı Külürr ${ }^{3}$ \\ ${ }^{1}$ Yıldız Technical University, Department of Geomatic Engineering, Division of Photogrammetry, Davutpasa Campus, \\ Esenler, 34210, Istanbul,TR \\ ${ }^{2}$ IDEGIS Technology, Information and Software Ltd. Company, Y1ldız Technical University Davutpasa Campus, \\ Technopark, Davutpasa Str, K-118, 34210 Esenler-/Istanbul-TR \\ ${ }^{3}$ Istanbul Technical University, Department of Geomatics Engineering, 34469 Maslak Istanbul, Turkey \\ *Corresponding author \\ Tel : : +902123835329 \\ Received: 13 April 2017 \\ E-mail : bayram@ytu.edu.tr \\ Accepted: 03 May 2017
}

\begin{abstract}
The human face includes different colors and forms due to its complexity. Therefore, facial image processing comprises even more problems than image processing of other objects. Interest point detection is one of the important problems in computer vision, which is the key aspect of solving problems such as facial expression analysis, age analysis, sex defining, facial recognition, and three-dimensional face modelling in augmented reality. To accomplish these tasks, facial interest points need automatic definition. A hybrid algorithm was developed to detect automatically interest regions and points in multiple images in the resented study. The study used processed facial images from an authorized image database with a resolution of $1600 \times 1200$, taken in standardized illumination conditions by using an InSpeck Mega Capturor II optical 3D structured light digitizer and 1000-W halogen lamp. The presented study integrated skin color analysis with the Haar classification method, processing 11 male and 25 female facial images with the developed algorithm. The average accuracy of facial interest point detection was $0.68 \mathrm{~mm}$ after testing all images.
\end{abstract}

Keywords: Close-range photogrammetry, face recognition and facial interest points, image matching and processing

\section{Introduction}

Automatic recognition of human faces and detection of sensory organs is one of the most popular research topics in recent years (Harmon, 1977; Samal and Iyengar, 1992; Valentin et al., 1994; Xiaoping, 2011; Bansal, 2012; Găianua and Onchiş, 2014). A system that performs face detection or recognition will find many applications such as surveillance cameras and security control systems (Kondo and Yan, 1999). Face recognition and expression analysis algorithms have received most of the attention in the academic literature in comparison to face detection (Delakis and Garcia, 2002). Due to homogeneous structure of the face, detection of facial interest points, 3D face modelling, and emotion analysis are challenging tasks, and are still the focus of many researchers (Koo and Song, 2010; Ma, 2011; Valstar, 2011). Image matching is an important aspect of the 3D face modelling process. The two main components of image matching are selection of matching units and similarity measurement. A matching unit is a group of details that are compared in multiple stereo images. Similarity measurement gives information about the matching conditions of matched units. Image matching uses interest points to define and distinguish the objects in some feature-based methods. Face recognition, detection, and algorithms for defining facial interest region points can be affected by many components, such as changes of facial emotion, illumination conditions, photographing angle, and distance between object and camera (Huorong et al., 2014; Saha and Bhattacharjee, 2012).

Research of Viola and Jones (2001) which is based on facial detection rapid key feature classifier developing is accepted as the pioneer study by many researchers. While some developed algorithms work with different data sets, others use restricted data sets to achieve more rapid solutions or detect specific points on the face (Saha and Bhattacharjee, 2012). The interest points have to be located precisely 
(Eser, 2006), especially in biometric methods, which are used for security purposes in automatic identity definition systems.

The developed techniques on the topic of automatic interest point detection can be classified as geometry- and symmetry-based, template-based, color-based, and appearancebased techniques (Brunelli and Poggio, 1993; Bhownik et al., 2013). In color-based techniques, pre-processing of facial images is usually required due to unstandardized illumination (Bhumika and Zankhana, 2011). Modelling of skin color in the $\mathrm{YCbCr}$ color space is one of the suggested methods to overcome this problem (Chai and Ngan 1999). Template-based techniques are semi-automatic methods, and facial interest operators are measured manually (Lee and Thalmann, 2011).

The detection of facial interest points is not generally required in appearance-based techniques, but is used commonly for facedetection purposes (Brunelli and Poggio, 1993). Since these methods by themselves are not effective for some tasks, hybrid methods have been developed to generate precise, more successful results by using combinations of these techniques (Huorong, et al., 2014; Reinders et al., 1995; Sobottka and Pitas, 1996; Fröba and Küblbeck 2001; Tian and Bolle2001; Feris, et al., 2002).

The present study used facial images that were taken with a stable-positioned camera in three different perspectives - left profile, frontal, and right profile-with each stereo pair having $80 \%$ overlap. The developed algorithm first detects interest regions, then within these regions detects intelligent interest points, and by using these points, matches all three facial images. The developed method was designed to detect interest points and to match images in both profile and frontal images. In addition, the algorithm can automatically find the location and region of any interest points on the face, find which interest point belongs to which sensory organ, and determine the direction of all interest points. As a result, interest regions and points can be defined with greater sensitivity to the photographing angle. The presented study was developed on the .NET platform and coded in the $\mathrm{C}++$ and $\mathrm{CH}$ programming languages. The $\mathrm{OpenCv}$ open 117 source library (2012) used for the main graphical processes and the URL 1 (2012) open source image-processing library was used for image processing.

\section{Material and Method}

Humans could identify faces in a scene with their natural abilities without any additional equipment. It is very difficult to create an automated system for the identification task (Samal and Iyengar, 1992). The developments in hardware and software of computer technologies are removing the limit of the difficulty. The problem of finding face patterns is actual problematic due to the large variation of distortions that have to take into reason. These distortions include different facial expressions, environmental conditions, perspective of view. After any trial of physically enumerating every possible situation, we can easily conclude that this procedure is endless (Delakis and Garcia, 2002). Many facial interest region and point detection-related studies were realized by using different standard data sets (Huorong et al., 2014; Eser, 2006; Demirel and Anbarjafari 2008; Ar, 2008; URL 2, 2011; URL 3, 2012).

The Bosphorus database of Bosphorus University, Turkey, and its facial data sets were used in the presented study (Savran et al., 2008). The facial images in the Bosphorus database were taken with an InSpeck Mega Capturor II optical 3D structured light digitizer. The spatial resolution of the instrument is 0.3 $\mathrm{mm}$ in the x-axis, $0.3 \mathrm{~mm}$ in the $\mathrm{y}$-axis, and 0.4 $\mathrm{mm}$ in the z-axis. The resolution of RGB images is $1600 \times 1200$. A $1000 \mathrm{~W}$ halogen lamp was used to obtain homogeneous illumination and to reduce noise during photographing.

This proposed study consists of six main steps: data pre-processing, face detection, defining of interest regions, determining of interest points, detecting, and image matching. If no preprocessing, or image enhancement, was required, the algorithm starts with the second step, face detection. The algorithm automatically defines the direction of images (left profile, frontal, or right profile) with a histogram analysis of skin color. In the third step, interest regions can be defined after 
analysing facial images and fixing which interest regions are included in the images. In the fourth step, special key points are searched and correlated with each interest region. As a result, interest points can be defined precisely in the fifth step. The achieved interest points are used for image matching in the last step.

\section{Face Detection}

In this study, classifiers for face detection are integrated with skin color filtering methods. Obtained test results indicate that the ViolaJones 9 method did not give very satisfying results with used data set; in particular, results with profile images were ineffective. Thus, skin color analysis was integrated with the Haar classification method to detect interest regions, especially in profile images. The face detection step was achieved through using the following face geometry rules:

- Interest regions were ordered, from top to bottom, as eyebrow-eye-nose-lip.

- $\quad$ Two eyebrows and eyes, one nose, and one lip interest region have to be found in frontal images.

- The nose region between two eyebrow-eye regions must cover less area than the lip region in frontal images.

- Only one of the interest regions (eyebrow, eye, nose, lip) has to be found in profile images.
The nose region is at the left or right of both eyebrow-eye regions, according to the direction of the profile, and covers less area than the lip region.

The interest regions were searched in all images, and for each image, the face/not face decision was realized. The critical problem with skin color filtering is choosing the color space (Eser, 2006; Shin et al., 2002; Kim et al., 2003). Recently, studies on this topic have suggested that applying HSV and YCbCr (Poynton, 1985) color spaces together revealed considerably more accurate results (Kurt, 2007). In the presented study, HSV color space is used for basic skin color analysis, while RGB, HSV, and $\mathrm{YCbCr}$ color spaces are used for detailed skin color analysis to separate interest regions properly in facial images. The empirical threshold values are defined as follows:

$$
(\mathrm{H}<18, \mathrm{~S}<50, \mathrm{~V}<80) .
$$

Detailed skin color analysis was obtained by applying hierarchic rules. The rule sets were defined for RGB color space, YCbCr color space, and HSV color space. Filtering results were obtained after applying the rules for each color space. The rules are defined as follows (Çavdaroğlu, 2013).

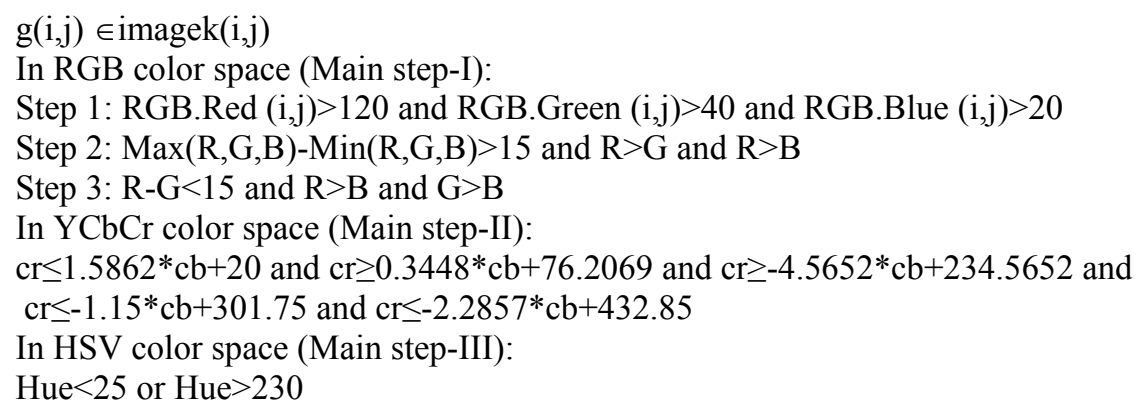

Following integration of the Viola-Jones (2001) and skin color filtering methods, the connected components labelling method was applied (Rosenfeld, 1970; Rosenfeld and Kak, 1976) to obtain blobs. Faces and interest regions in the faces were detected as independent blobs. The blob with the largest area, and included facial interest regions, was defined as a facial component.

\section{Defining of frontal/profile poses by histogram analysis}

Defining interest regions in the facial area involves the problems of the interest regions and the count of the regions that have to be searched, which has been solved. For example, in frontal images, interest regions on both sides 
of the face are used. However, interest regions in only one direction have to be used in left or right profile images. Therefore, the direction of the image must be defined first, and then the interest regions. By analysing the horizontal histogram, the direction of images is determined. By analysing the vertical histogram, which interest regions have to be searched in an image is determined.

Horizontal and vertical histograms have been calculated from the created binary facial images. Since white pixels belong to the skin, and black pixels to the rest of the facial tissue in the binarized image, horizontal and vertical histograms were obtained from the sum of the pixel values along rows and columns, and the analysis of the histograms was done in pixel units. Due to the horizontal and vertical pixel numbers of all binarized facial regions, the sums of the row pixels were taken into account regardless of whether the obtained image is a frontal or profile pose. This enabled us to pinpoint the locations of $\mathrm{x}$ - and $\mathrm{y}$-pixel coordinates that correspond to the histogram, and accordingly, the locations of related sense organs in the vertical and horizontal histogram analyses. In the horizontal histogram, local minimum and maximum points emerge depending on whether the image was a frontal or profile pose, while in the vertical histogram, it depends on the number of interest regions that the image covers. The analysis of the local maximum and minimum points help determine the type of imaging, thereby making it possible to identify the number and type of correlation zones on the image. Fig. 1 illustrates the vertical and horizontal histograms of a sample image and the local minimum and maximum spots.

The expected local minimum and maximum point numbers in the histogram are given. With the horizontal histogram analysis, it is identified whether an image is a frontal or a profile pose and by the vertical histogram analysis, correlation zones in this facial image which are going to be searched are determined. During the frontal/profile pose identification, the rules derived from the symmetry pattern of facial geometry were applied to the distribution of skin color pixels in the face. Depending on this information in the horizontal histogram analysis, it was decided that at least one and at most two minimum points, and two or three maximum interest points, are created.

As seen in Fig. 1a, 1b, and 1c, the top two zones of the histogram distribution are smaller than the zone that appears in Fig. 1,a. In the vertical histogram analysis, it was detected that four minimum points are needed, as sense organs in the frontal face image come in four different types. Sequential corrections in the horizontal and vertical histogram analyses were maintained until the target minimum and maximum point numbers in the face histogram distribution were reached.
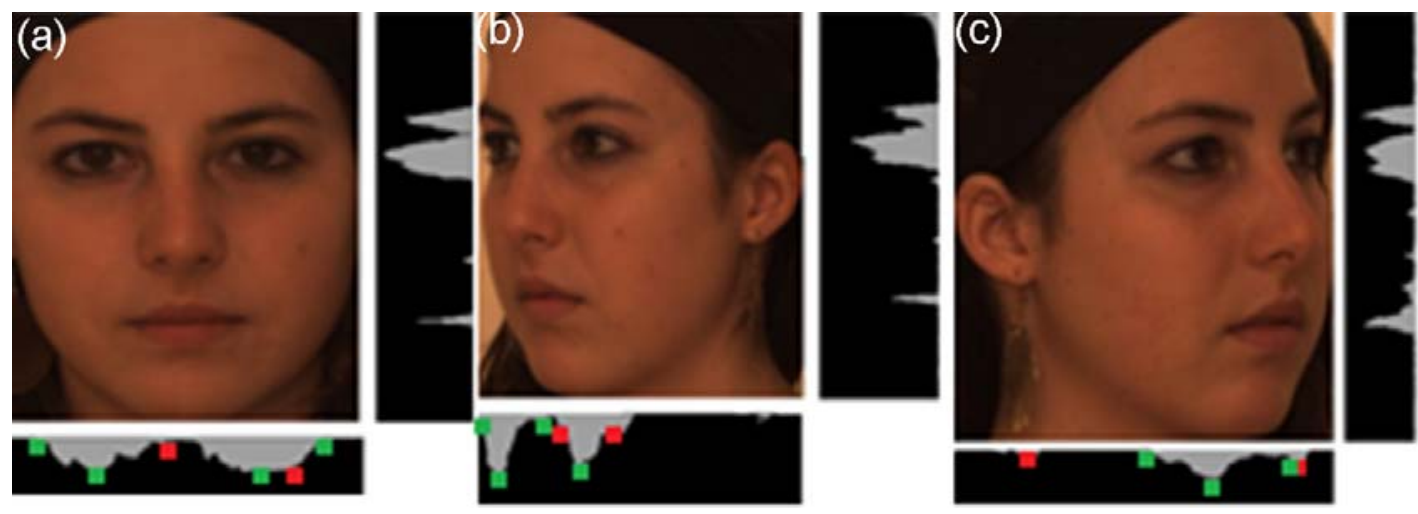

Fig 1a. Horizontal and vertical histogram distribution of sample frontal image. b. Horizontal and vertical histogram distribution of sample left profile image. c. Horizontal and vertical histogram distribution of sample right profile image. 


\section{Identification of interest zones and points}

In the phase of face recognition, the temporal image results obtained by skin colour filtering were utilized in order to identify interest regions. Since in the histogram analysis, the localization of the interest regions in the face was defined, this information was used in the identification step. Thus, undefined interest regions were segmented according to their region type and location on the face. The convex polygon, which surrounds the independent component marked as the facial zone, was formed, and others which have been
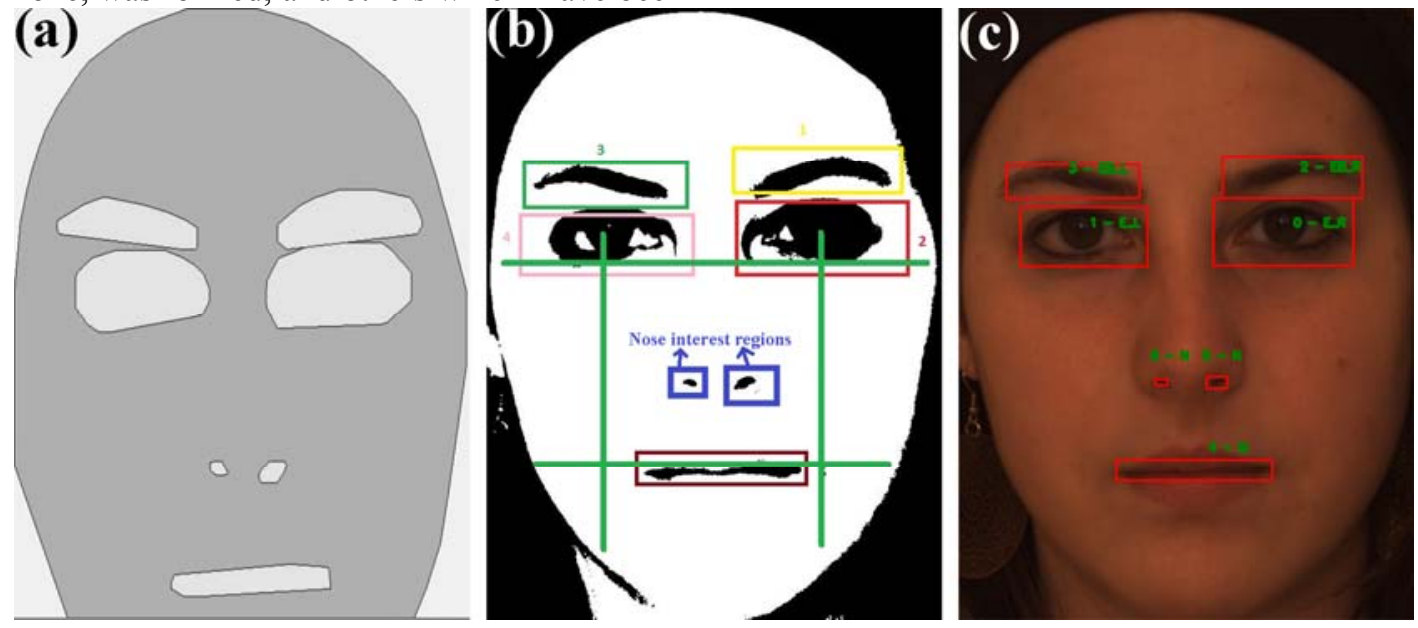

Fig 2a. Sample face sketch, b. Merging nose-interest regions, c. Identified interest regions
The middle points of the two eyebrows and eye regions were calculated; the middle points of nose and lip interest regions are estimated to be between these points. The rule for left/right profile poses is that the middle points of nose and lip interest regions are in the right side of the middle points of eyebrow/eye interest regions in right profile poses, and in the left side of the middle points of eyebrow/eye interest regions in left profile poses. The bottommost region was identified as the lip interest region. If the lip interest regions can be segmented in pieces, they should be merged. For this process, the pieces in the same row number in the bottommost of the facial image were merged. Following this step, the regions between two eye regions horizontally and between the eye and lip regions vertically were merged as the nose region (Fig. 2b). The identified regions are given in Fig. 2c. found were filtered depending on whether they remain within the face's convex polygon or not. Following the rough identification of interest regions, definitions were made for each zone according to the location of the interest region on the face and its proximity to other interest regions through using face geometry. The sample face sketch is given in Fig. 2a. To identify the interest regions, two eyebrow and eye interest regions were searched in frontal images, while one eyebrow and eye interest region was searched in right-left profile images.
The interest points were identified with the interest point operators which have been developed in this study. A separate operator was also developed for each facial interest zone (eyebrows, eyes, nose, and lips). In this way, as the interest point operators knew about the distinguishing features of the points they were to look for, the interest points in the zone were identified by making use of this information.

For eyebrow, eye, lip, and nose interest-point detection, the same histogram analysis was used. The interest region analyses consist of four main steps:

(i) Interest-point operator uses related interest region.

(ii) Binary image of the interest region is created.

(iii) The horizontal and vertical histograms are created, defined as in the previous sub section. 
(iv) Horizontal and vertical histograms are analysed and interest points are marked; in other words, their image coordinates are obtained.

\section{Eyebrow interest points}

First, the pixels in the region were filtered, RGB values were converted to $\mathrm{YCbCr}$, and the average of the $\mathrm{Cr}$ component was saved. This value was used for thresholding of the original image in the interest region. The vertical histogram were then created. In the vertical histogram analysis, the $x-y$ coordinates that correspond to the lowest histogram height were defined as the middle point of the eyebrow. This point splits the histogram into two regions. The first bar of the histogram in the left region was marked as the start of the eyebrow, and the last bar of the histogram in the right region was marked as the end of the eyebrow (Fig. 3).

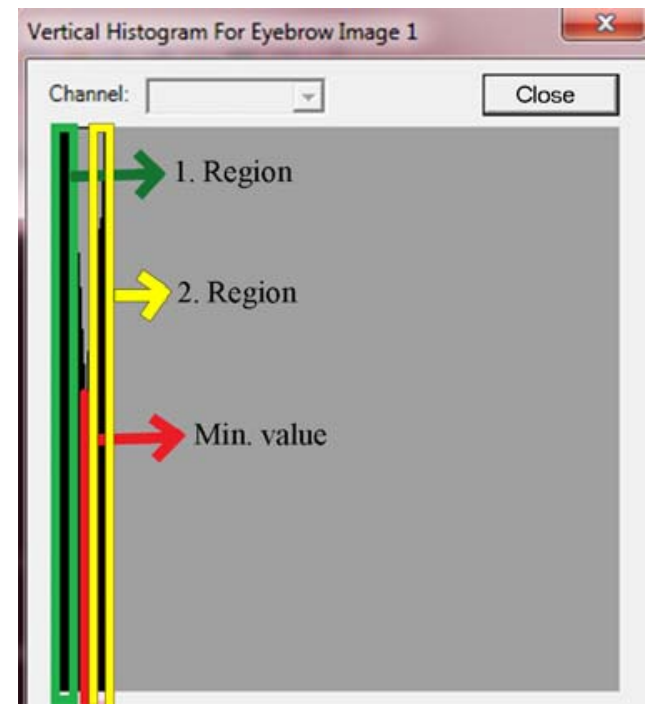

Fig. 3 Vertical histogram for detecting eyebrow interest points

\section{Eye interest points}

Similar to the eyebrow interest-point operator, the average $\mathrm{Cr}$ component was calculated for the eye interest region and the binary image was created. In the original image patch, which overlaps with the binary image, for each row $\mathrm{Y}$, $\mathrm{Cr}$ components of $\mathrm{YCbCr}$ colours were used, an average value was calculated, and another binary image was created. Following this step, vertical and horizontal histogram analyses were carried out. The maximum value of the horizontal histogram corresponds to the $\mathrm{x}$-value of the iris point, while the maximum value of the vertical histogram corresponds to the $y$ value of the iris point. The minimum values of the left and right side of the iris in the vertical histogram were marked as the left and right interest points of the eye, and the minimum values of the left and right side of the iris in the horizontal histogram were marked as the bottom and top interest points of the eye (Fig. 4).

\section{Lip interest points}

The lip interest-point operator uses the lip interest region and analysis horizontal and vertical histograms. Due to the large grey value difference of lip pixels compared to skin pixels, it was possible to distinguish the lip pixels by applying detailed skin colour analysis in this step. Thus, a binary image was created which includes only the segmented lip region (Fig. 5). The lip contours were obtained by applying connected components analysis. The line passing through the midpoint of the convex polygon overlaps with the lip's middle line. To define the start and end points of the lip, the maximum histogram value was first found. This line splits the histogram into two regions. The point at which its y-value is equal with the lip main line and its $\mathrm{x}$-value corresponds to the minimum histogram high in the left region was marked as the start point of the lip, and the point where its $\mathrm{x}$-value corresponds to the maximum histogram high in the right region was marked as the end point of the lip. Similarly, the upper and bottom interest points were found by using the vertical histogram of the interest region. 


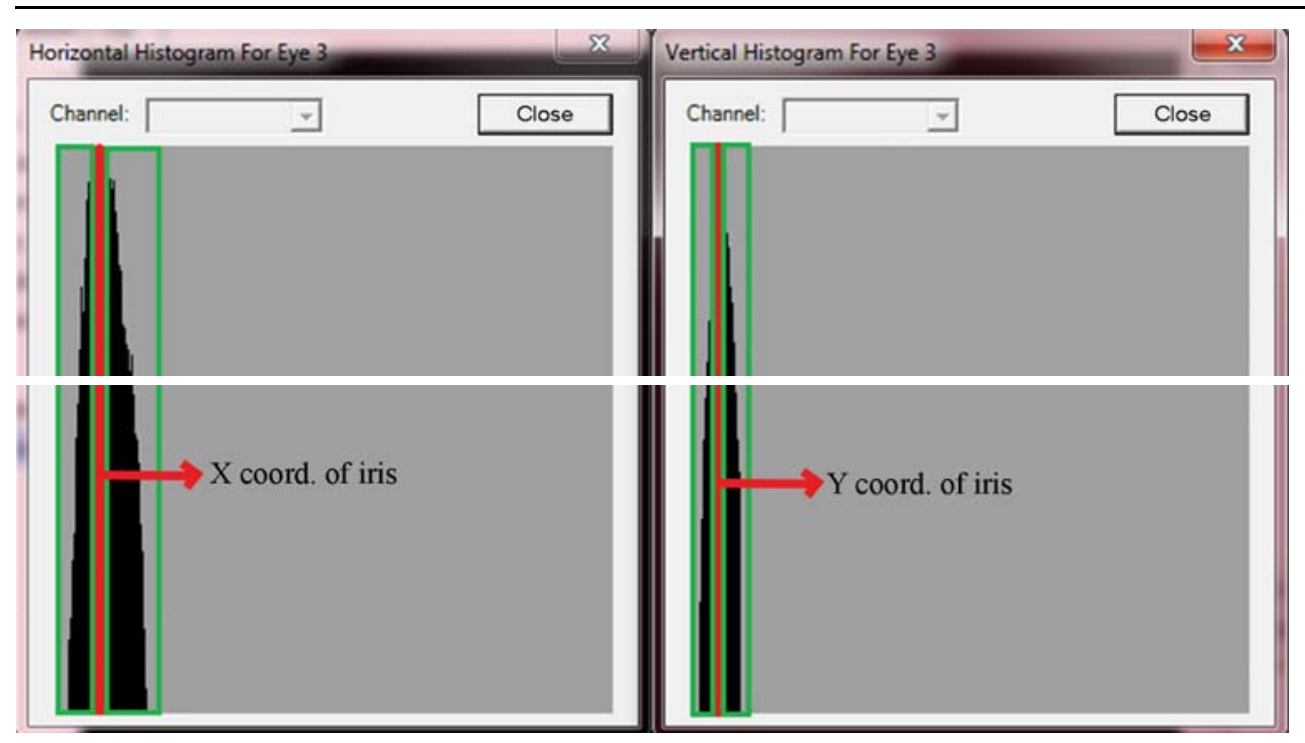

Fig 4. Vertical and horizontal histograms for detecting eye interest points

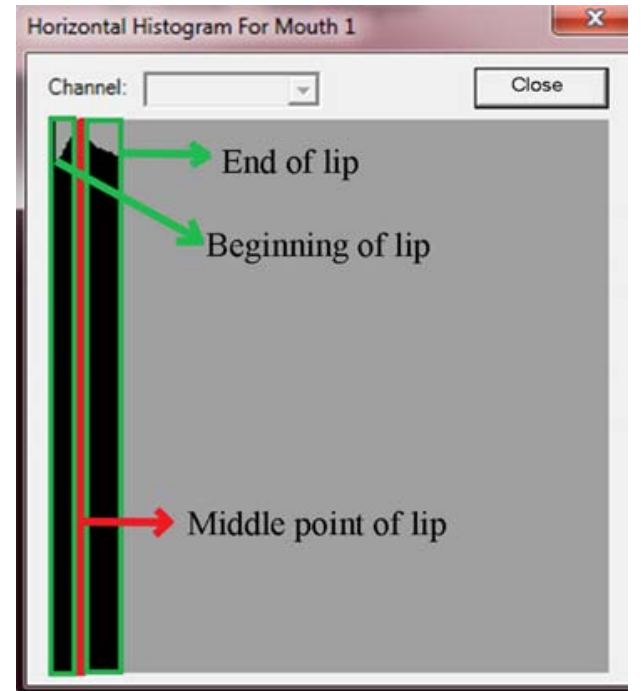

Fig 5 Horizontal histogram for detecting lip interest points

\section{Nose interest points}

The nose interest-point operator uses the nose interest region and analysis horizontal and vertical histograms. This operator creates two different horizontal histograms and one vertical histogram. The nose holes are darker than the skin colour, so they were obtained and binarized easily by applying skin colour analysis and connected components analysis.
The $\mathrm{Cr}$ component of each pixel in the $\mathrm{YCbCr}$ colour space and L (luminance) component of each pixel in the HSL colour space were used to create two horizontal histograms. The vertical histogram was created by using the $\mathrm{Cr}$ component.

The maximum value in the first histogram splits the histogram into two regions (Fig. 6,a). The minimum value in the second histogram represents the $\mathrm{x}$-coordinate of the middle point of the nose (Fig. 6,b). The minimum value of both left and right regions corresponds to the edge points of the nose holes. Similarly, the maximum and minimum values of the vertical histogram were found. The region between the maximum and minimum values was analysed, and the maximum value inside of the region was marked as the y-coordinate of the middle point of the nose (Fig. 6,c).

Once all the steps were completed, the face was identified and located in the input image, and the interest regions and points of each interest region were identified and defined. After definition of the interest points for all stereo pairs, the image matching process can be accomplished easily. 


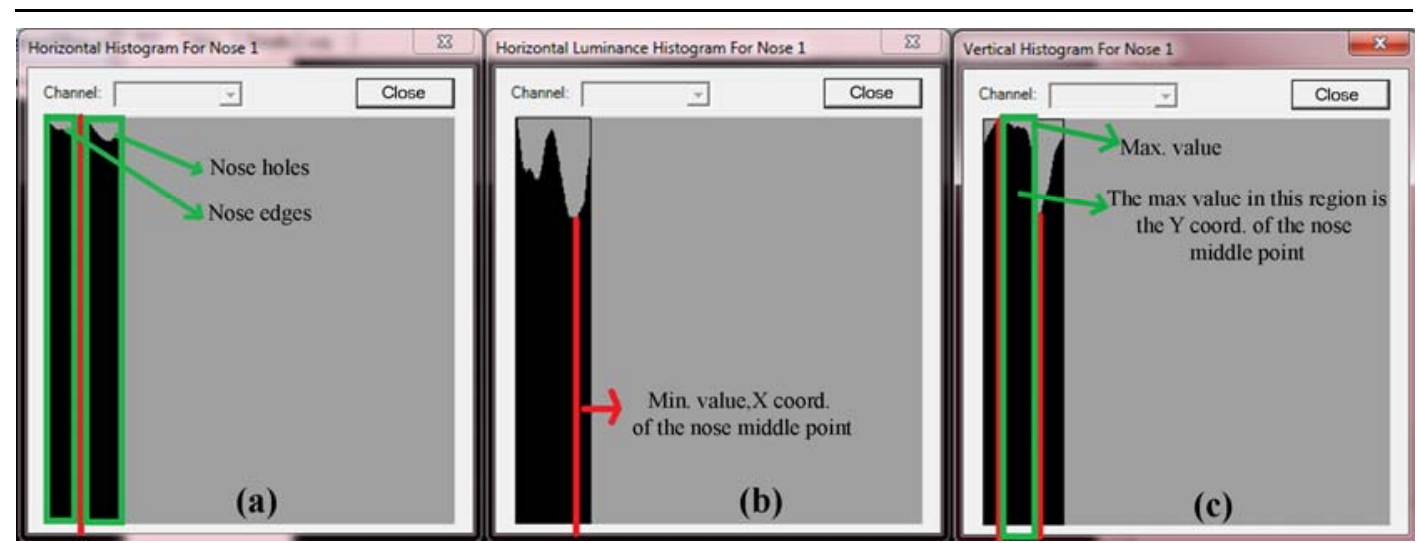

Fig 6a. Horizontal histogram for detecting nose interest points. b. Horizontal luminance histogram for detecting nose interest points. c. Vertical histogram for detecting nose interest points.
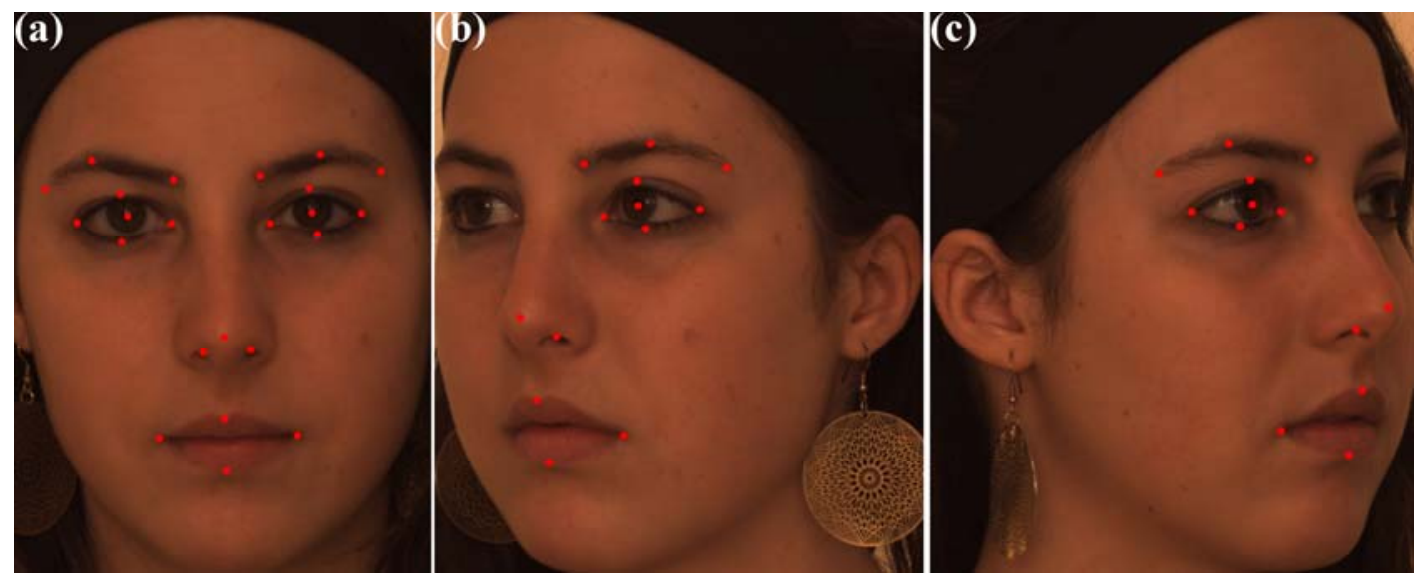

Fig7a. Interest points on frontal image. b. Interest points on left profile image. c. Interest points on right profile image

\section{Results}

With the interest-point operators detected by developed algorithm, the overall accuracy was calculated as $0.68 \mathrm{~mm}$ for 10 test images. The calculation was done by comparing interest points between the results of the proposed algorithm and the Bosphorus database.

Interest-point operators provide the horizontal and vertical interest histogram statistics according to the RGB, HSV, and $\mathrm{YCbCr}$ values in the original image, and obtain the points from these statistics. Primarily, four interestpoint operators work to create interest points related to their interest regions: eyebrow, eye, nose, and lip. Fig. 7a-c illustrates the results obtained by the application of the process of interest-point identification on frontal, left profile, and right profile images, respectively.
Ten different test images and fifteen interest points were used for the accuracy assessment. The comparison was performed by calculating the coordinate difference between the detected points and the manually labelled points in the Bosphorus Database. Table 1 illustrates the accuracies' interest-point detection obtained from the sample data. In the table 1,1 . interest point represents the outer left eyebrow, 2 . middle left eyebrow, 3. inner left eyebrow, 4. inner right eyebrow, 5. middle right eyebrow, 6 . outer right eyebrow, 7 . outer left eye corner, 8 . inner left eye corner, 9. inner right eye corner, 10. outer right eye corner, 11. nose tip, 12. left mouth corner, 13. upper lip outer middle, 14. right mouth corner and 15 is the lower lip outer middle.

By the help of intelligent interest points, the necessity to operate the identifiers that run the process of searching for a point in a stereo pair 
image was eliminated, and it became possible to find stereo pair correspondents of the points in the stereo pair image with the same developed algorithm in this study. The search for a point in facial images that comprise similar quality pixels results in both prolonged Table 1 Comparison the obtained results with Bosphorus Database

\begin{tabular}{|c|c|c|c|c|c|c|c|c|c|c|c|}
\hline Face no & $\begin{array}{c}\mathrm{BS} 2 \\
6\end{array}$ & $\begin{array}{c}\text { BS3 } \\
1\end{array}$ & $\begin{array}{c}\mathrm{BS} 2 \\
9\end{array}$ & $\begin{array}{c}\text { BS4 } \\
5\end{array}$ & $\begin{array}{c}\text { BS5 } \\
2\end{array}$ & $\begin{array}{c}\text { BS6 } \\
9\end{array}$ & $\begin{array}{c}\text { BS7 } \\
1\end{array}$ & $\begin{array}{c}\text { BS7 } \\
4\end{array}$ & $\begin{array}{c}\text { BS8 } \\
3\end{array}$ & $\begin{array}{c}\text { BS9 } \\
6\end{array}$ & \multirow{3}{*}{$\begin{array}{c}\text { MEA } \\
\mathbf{N} \\
0,44 \\
\end{array}$} \\
\hline Int. Point no & \multicolumn{10}{|c|}{ differences in $\mathrm{mm}$} & \\
\hline 1 & 0,22 & 0,58 & 0,22 & 0,47 & 0,42 & 1,06 & 0,41 & 0,34 & 0,21 & 0,46 & \\
\hline 2 & 0,44 & 0,42 & 0,60 & 0,61 & 0,64 & 0,88 & 0,47 & 0,39 & 0,45 & 0,65 & 0,55 \\
\hline 3 & 0,17 & 0,18 & 0,31 & 0,70 & 0,32 & 1,07 & 1,54 & 1,27 & 0,65 & 1,67 & 0,79 \\
\hline 4 & 0,34 & 0,74 & 0,44 & 0,66 & 0,66 & 1,34 & 0,77 & 0,23 & 0,72 & 1,06 & 0,70 \\
\hline 5 & 1,04 & 0,40 & 0,36 & 0,71 & 0,64 & 1,27 & 0,79 & 0,71 & 0,16 & 0,81 & 0,69 \\
\hline 6 & 0,35 & 0,73 & 0,75 & 0,99 & 0,84 & 0,68 & 0,45 & 0,39 & 0,70 & 0,84 & 0,67 \\
\hline 7 & 0,21 & 0,69 & 0,56 & 0,91 & 0,63 & 1,15 & 0,75 & 0,76 & 0,67 & 1,00 & 0,73 \\
\hline 8 & 1,60 & 0,42 & 0,26 & 0,55 & 0,64 & 0,58 & 0,21 & 0,61 & 0,53 & 0,57 & 0,60 \\
\hline 9 & 1,53 & 0,74 & 0,92 & 0,66 & 0,08 & 0,56 & 1,17 & 1,26 & 0,75 & 1,39 & 0,91 \\
\hline 10 & 0,42 & 0,55 & 0,91 & 0,59 & 0,84 & 1,35 & 0,87 & 0,90 & 0,53 & 1,02 & 0,80 \\
\hline 11 & 0,32 & 0,33 & 0,81 & 0,22 & 0,28 & 1,05 & 0,20 & 0,53 & 0,59 & 0,63 & 0,50 \\
\hline 12 & 0,43 & 1,62 & 0,76 & 0,91 & 0,39 & 0,86 & 0,31 & 1,13 & 0,82 & 0,88 & 0,81 \\
\hline 13 & 0,83 & 0,64 & 0,46 & 0,18 & 0,75 & 0,70 & 0,24 & 0,69 & 0,56 & 0,61 & 0,57 \\
\hline 14 & 0,71 & 0,88 & 1,09 & 0,63 & 0,59 & 0,95 & 1,01 & 1,62 & 0,22 & 1,03 & 0,87 \\
\hline 15 & 0,29 & 0,64 & 0,36 & 1,06 & 0,27 & 0,58 & 0,27 & 0,83 & 0,60 & 0,66 & 0,55 \\
\hline Overall & 0,59 & 0,64 & 0,59 & 0,66 & 0,53 & 0,94 & 0,63 & 0,78 & 0,54 & 0,88 & 0,68 \\
\hline
\end{tabular}

\section{Discussion}

The algorithm was compared with well-known interest operators such as Harris (Rosenfeld and Kak, 1976), Surf (Davies, 2012) and Fast (Jazayeri and Fraser, 2010). As these operators were developed to serve general purposes, when special kinds of data that have such small sizes as the human face are to be studied, there emerges a need to develop operators that are appropriate for the data. Fig. 8 illustrates the results obtained as an output of the application of some recognized operators on three test images and different results for the first image for different operators have been evaluated and obtained results were displayed in Fig. 8.

With the Harris operator, two points on the right ear were identified Fig. 8a. With the Fast seek-outs and inaccurate matches due to the similarities. The identification of interest points defined in each stereo image removes the need for the matching process, and makes it possible to match the points by using their definitions 
The algorithm developed in this study runs properly on profile images taken, on average, from a 45-degree angle; however, accuracy ratios declined as the angle of the profile image approaches 90 degrees.

The points found by the developed system can be used for the matching of images that stand in
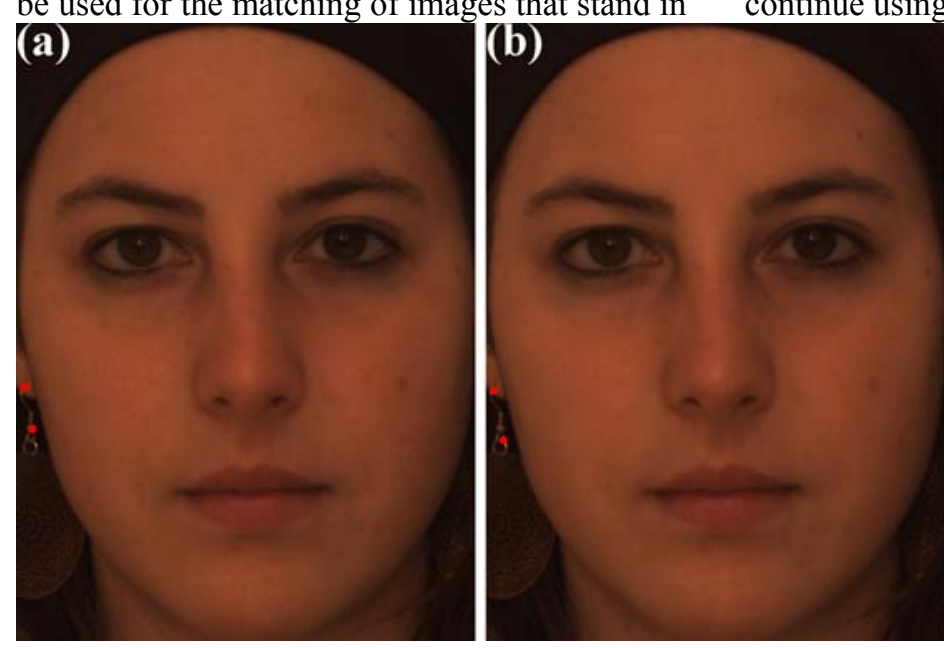

an appropriate range of angles. In this way, the matching process is facilitated in facial data comprised of points that have very similar features.

In the future, flexibility of the proposed algorithm will be improved and research will continue using different face databases.

Fig 8a. The application of Harris operator on test data. b. The application of Fast operator on test data. c. The application of Surf operator on test data.

\section{References}

Ar, I. (2008). Recognition of Human Behaviours In Video Streams. Seminar report, Gebze Institute of Technology, Dept. of Informatics, Izmit-Turkey.

Bansal, A. (2012). Face recognition using PCA and LDA Algorithm. In: Advanced Computing and Communication Technologies (ACCT), Second International Conference. Rohtak- India, 251-254.

Bhowmik, M.K., Shil, S. and Saha, P. (2013). Feature Points Extraction of Thermal Face using Harris Interest Point Detection. In: International Conference on Computational Intelligence: Modeling Techniques and Applications (CIMTA), University of Kalyani. West Bengal, India, September 2728 2013, Procedia Technology 2013; 10: $724-730$.

Bhumika, G. and Zankhana, H. (2011). Face Feature Extraction Techniques: A Survey. In: National Conference on Recent Trends in Engineering \& Technology. Anand, Gujarat, India, 13-14 May 2011.

Brunelli, R. and Poggio, T. (1993). Face Recognition: Features Versus Templates,
IEEE Transactions on PAMI, 15(10), 10421052.

Çavdaroğlu, G.Ç. (2013). Face Recognition Analysis by Developing Feature Operators In Virtual Reality, MSc Thesis, Yildiz Technical University, Institute of Natural and Applied Sciences, Istanbul-Turkey.

Chai, D. and Ngan, K. (1999). Face Segmentation Using Skin Color Map in Videophone Application. Transactions on Circuits and Systems for Video Technology, 9(4): 551-564.

Davies, E.R. (2012). Corner and Interest Point Detection, Computer and Machine Vision (Fourt Edition), Chapter 6: 149-184.

Delakis, M. and Garcia, C. (2002). Robust Face Detection Based on Convolutional Neural Networks, 2nd Hellenic Conf. on Al., SETN-2002, 11-12 April 2002, Thessaloniki, Gereece, Proceesing: 367378.

Demirel, H. and Anbarjafari, G. (2008). A New Face Recognition System Based On Color Histogram Matching. In: Signal Processing, Communication and Applications Conference, SIU, 22-28 April, Aydin, Turkey: 1-4. 
Eser, S. (2006). Face Recognition And Tracking By Neural Networks, MS Thesis, Yildiz Technical University, Institute of Natural and Applied Sciences, IstanbulTurkey.

Feris, R.S., Gemmell, J., Toyama, K. and Krüger, V. (2002). Hierarchical Wavelet Networks for Facial Feature Localization. In: Proceedings of the 5th International Conference on Automatic Face and Gesture Recognition. Washington D.C. USA, 21 May, 2002: 118-123.

Fröba, B. and Küblbeck, C. (2001). Real-Time Face Detection Using Edge-Orientation Matching. In: Third International Conference, AVBPA, Lecture Notes in Computer Science, Halmstad, Sweden, 6-8 June 2001, 2091: 78-83.

Găianua, M. and Onchiş, D.M. (2014). Face and Marker Detection Using Gabor Frames on GPUs. Signal Processing, 96: 90-93.

Harmon, L.D. (1977). Automatic recognition of human face profiles, Computer Graphics and Image Processing, 6 (2):135-156.

Jazayeri, I. and Fraser, C. (2010). Interest Operators For Feature - Based Matching In Close Range Photogrammetry, The Photogrammetric Record, 25(129): 24-41.

Kim, I., Shim, H.J. and Yang, J. (2003). Face detection, Face Detection Project, EE368, Stanford University, Vol. 28.

Kondo, T. and Yan, H. (1999). Automatic human face detection and recognition under non-uniform illumination, Pattern Recognition, 32: 1707-1718.

Koo, H. and Song, H. (2010). Facial Feature Extraction for Face Modeling Program. Int. J. Circuits, 4(4): 169-176.

Kurt, B. (2007). Physiological State Evaluation, MSc Thesis, Black Sea Technical University, Institute of Natural and Applied Sciences, Trabzon-Turkey.

Lee, S. and Thalmann, N. (2011). Fast Head Modeling For Animation. Image Vis. Comput., 14 (4): 355-364.

Ma, T.C. (2011). 3D Facial Reconstruction System from Skull for Vietnamese. Knowledge and Systems Engineering (KSE) In: Third International Conference. HanoVietnam, 14-17 October 2011: 120-127.

Poynton, C. A. (1985). Guided Tour Of Color Space. New Foundations for video Technology, In: Proceedings of the SMTPE
Advanced Television and Electronic Imaging Conference. San Francisco, USA, Feb. 10-11: 167-180.

Reinders, M.J.T., Van Beek, P.J.L., Sankur, B. and Van Der Lubbe, J.C.A. (1995). Facial Feature Localization and Adaptation of A Generic Face Model For Model-Based Coding. Signal Processing: Image Communication, 7: 57-74.

Huorong, R., Jianwei, S., Yanhong, H., Xinxin, Y. and Yang, L. (2014). Uniform Local Derivative Patterns and Their Application in Face Recognition. Journal of Signal Processing Systems, 74 (3): 405-416.

Rosenfeld, A. (1970). A Connectivity in Digital Pictures. Journal of the ACM (JACM), 17(1): 146-160.

Rosenfeld, A. and Kak, A.C. (1976). Digital Picture Processing, New York, Sec 81, Academic Press.

Saha, R. and Bhattacharjee, D. (2012). Memory Efficient Human Face Recognition Using Fiducial Points. Int. J. of Adv. Res. in Comput. Sci. and Softw. Eng. 2(1):1-8

Samal, A. and Iyengar, P.A. (1992). Automatic recognition and analysis of human faces and facial expressions: a survey, Pattern Recognition, 25 (1): 65-77.

Savran,N. A., Dibeklioğlu, H., Çeliktutan, O., Gökberk, B. , Sankur, B. and Akarun,L. (2008). Bosphorus Database for 3D Face Analysis. In: The First COST 2101 Biometrics and Identity Management: Workshop on Biometrics and Identity Management (BIOID 2008). Roskilde University, Denmark, 7-9 May, Revised Selected Papers, Lecture Notes in Computer Science, Volume 5372: 47-56.

Shin, M., Chang, K. and Tsap, L. (2002). Does Color Space Transformation Make Any Difference On Skin Detection? In: IEEE Workshop on Applications of Computer Vision. Orlando, FL, USA, 3-4 December ISBN 0-7695-1858-3: 275 - 279.

Sobottka, K. and Pitas, I. (1996). Extraction of Facial Regions and Features Using Color and Shape Information. In: Proc. of Int. Conf. on Pattern Recognition, 3: 421 - 425.

Tian,Y. and Bolle, R.M. (2001). Automatic Neutral Face Detection Using Location And Shape Features, Computer Science Research Report RC 22259, IBM Research.

URL 1 (2012). http://www.aforgenet.com/ 
URL 2 (2011). http://www.facerec.org/databases/

URL 3. (2012). http://www.facedetection.com

Valentin, D., Abdi, H., O'Toole, A.J. and Cottrell, G.W. (1994). Connectionist models of face processing: a survey, Pattern Recognition, 27: 1209-1230.

Valstar, M.F. (2011). The First Facial Expression Recognition and Analysis Challenge. In: Automatic Face \& Gesture Recognition and Workshops (FG 2011), IEEE International Conference. Santa Barbara, CA, USA, 21-25 March 2011: 921-926.

Viola, P. and Jones, M. (2001). Robust RealTime Object Detection. In: Second International Workshop On Statistical And Computational Theories Of Vision Modeling, Learning, Computing And Sampling. Vancouver, Canada, July 13, 2001.

Xiaoping, W. (2011). Face Recognition Based on Bionic Pattern. In: Electric Information and Control Engineering (ICEICE), 2011 International Conference, China, 15-17 April 2011: 2187 - 2189. 